\title{
Fabrication and Functionalization of Nanochannels by Electron-Beam-Induced Silicon Oxide Deposition ${ }^{\dagger}$
}

\author{
Christophe Danelon ${ }^{\ddagger}$ Christian Santschi ${ }^{\S, \|}$ Jürgen Brugger, ${ }^{\S}$ and Horst Vogel ${ }^{*, \ddagger}$ \\ Laboratory of Physical Chemistry of Polymers and Membranes, Ecole Polytechnique Fédérale de \\ Lausanne (EPFL), CH-1015 Lausanne, Switzerland, Microsystems Laboratory, EPFL, CH-1015 Lausanne, \\ Switzerland, and Centre Suisse d'Electronique et de Microtechnique (CSEM), \\ CH-2007 Neuchâtel, Switzerland
}

Received May 10, 2006. In Final Form: August 1, 2006

\begin{abstract}
We report on the fabrication and electrical characterization of functionalized solid-state nanopores in low stress silicon nitride membranes. First, a pore of $\sim 50 \mathrm{~nm}$ diameter was drilled using a focused ion beam technique, followed by the local deposition of silicon dioxide. A low-energy electron beam induced the decomposition of adsorbed tetraethyl orthosilicate resulting in site-selective functionalization of the nanopore by the formation of highly insulating silicon oxide. The deposition occurs monolayer by monolayer, which allows for control of the final diameter with subnanometer accuracy. Changes in the pore diameter could be monitored in real time by scanning electron microscopy. Recorded ion currents flowing through a single nanopore revealed asymmetry in the ion conduction properties with the sign of the applied potential. The low-frequency excess noise observed at negative voltage originated from stepwise conductance fluctuations of the open pore.
\end{abstract}

\section{Introduction}

Nanopore-based resistive-pulse sensors have been proposed for high-speed DNA sequencing:1,2 a single nanometer-sized pore fabricated in a thin, insulating, solid-state membrane mimics the function of a transmembrane protein channel in a lipid bilayer, thereby overcoming limitations in biological materials such as chemical, mechanical, and electrical instability. To achieve the required pore diameters in the range of a few nanometers, several approaches have been attempted, ${ }^{3-9}$ most of which take advantage of surface tension effects for tailoring the pore dimensions., ${ }^{4,9}$ Recently, Krapf et al. ${ }^{9}$ used the high-energy electron beam ( $>100$ $\mathrm{keV}$ ) of a transmission electron microscope (TEM) to drill 2.5 to $4 \mathrm{~nm}$ holes in $\mathrm{Si}_{x} \mathrm{~N}_{y}$ membrane. Broadening the beam increases the exposed area and reduces the intensity of the electron beam, which is accompanied by shrinking of the pores down to $2 \mathrm{~nm}$. Similarly, electron-beam-assisted shrinking has been observed in a silicon oxide membrane. ${ }^{6}$ Low-energy ion beam $(\sim 3 \mathrm{keV})$ irradiation of the proximity of prefabricated nanopores in a $\mathrm{Si}_{x} \mathrm{~N}_{y}$ membrane has also been used for size reduction below the ion beam diameter. ${ }^{4}$

Focused ion beam (FIB) is a versatile technique that allows the fabrication of structures ranging from a few tens of nanometers

\footnotetext{
† Part of the Electrochemistry special issue.

* To whom correspondence should be addressed. E-mail: horst.vogel@ epfl.ch.

† Ecole Polytechnique Fédérale de Lausanne (EPFL).

$\S$ Microsystems Laboratory.

"Centre Suisse d'Electronique et de Microtechnique (CSEM).

(1) Kasianowicz, J. J.; Brandin, E.; Branton, D.; Deamer, D. W. Proc. Natl. Acad. Sci. U.S.A. 1996, 93, 13770-13779.

(2) Deamer, D. W.; Akeson, M. Trends Biotechnol. 2000, 18, 147-151.

(3) Sun, L.; Crooks, R. M. J. Am. Chem. Soc. 2000, 122, 12340-12345.

(4) Li, J.; Stein. D.; McMullan, C.; Branton, D.; Aziz, M. J.; Golovchenko, J. A. Nature 2001, 412, 166-169.

(5) Siwy, Z.; Fulinski, A. Phys. Rev. Lett. 2002, 89, 198103.

(6) Storm, A. J.; Chen, J. H.; Ling, X. S.; Zandbergen, H. W.; Dekker, C. Nat. Mater. 2003, 2, 537-540.

(7) Saleh, O. A.; Sohn, L. L. Nano Lett. 2003, 3, 37-38

(8) Karhanek, M.; Kemp, J. T.; Pourmand, N.; Davis, R. W.; Webb, C. D. Nano Lett. 2005, 5, 403-407.

(9) Krapf, D.; Wu, M.-Y.; Smeets, R. M. M.; Zandbergen, H. W.; Dekker, C.; Lemay, S. G. Nano Lett. 2006, 6, 105-109.
}

to hundreds of micrometers. The fabrication of nanopores using FIB is inevitably associated with permanent surface charges due to ion implantation, which can inhibit the sensing abilities of the pore and increase electrical noise. The observed variability of the surface properties and the high cation selectivity of the pores make them unfavorable for precise analytical applications. ${ }^{10} \mathrm{In}$ the first approach to improvement, Branton and colleagues coated nanopore surfaces with metal oxides to produce functional nanopores with desirable electrical properties. ${ }^{10}$

Although the performance of fabricated nanopores has been demonstrated for single DNA molecule detection $7,8,11$ and size and folding identification, ${ }^{12-17}$ their application to nucleic acid sequencing requires single-base resolution, which still remains the major hurdle as linear polymers translocate too rapidly. Resolving DNA transport at the single-nucleotide level requires a considerable reduction of the diffusion rate of the molecule within the pore so that one base occupies the channel for at least 0.01 to $1 \mathrm{~ms} .{ }^{18}$ Inspired by recent experiments using engineered biological channels, ${ }^{19,20}$ nanopore surfaces can be modified with chemically reactive agents such as gold and silicon oxide, ${ }^{21,22}$

(10) Chen, P.; Mitsui, T.; Farmer, D. B.; Golovchenko, J.; Gordon, R. G.; Branton, D. Nano Lett. 2004, 4, 1333-1337.

(11) Chang, H.; Kosari, F.; Andreadakis, G.; Alam, M. A.; Vasmatzis, G.; Bashir, R. Nano Lett. 2004, 4, 1551-1556.

(12) Li, J.; Gershow, M.; Stein. D.; Brandin, E.; Golovchenko, J. A. Nat. Mater. 2003, 2, 611-615.

(13) Chen, P.; Gu, J.; Brandin, E.; Kim, Y.-R.; Wang, Q.; Branton, D. Nano Lett. 2004, 4, 2293-2298.

(14) Wang, H.; Dunning, J. E.; Huang, A. P.-H.; Nyamwanda, J. A.; Branton, D. Proc. Natl. Acad. Sci. U.S.A. 2004, 101, 13472-13477.

(15) Heng, J. B ; Ho, C.; Kim, T.; Timp, R ; Aksimentiev, A.; Grinkova, Y. V.; Sligar, S.; Schulten, K.; Timp, G. Biophys. J. 2004, 87, 2905-2911.

(16) Storm, A. J.; Chen, J. H.; Zandbergen, H. W.; Dekker, C. Phys. Rev. E 2005, 71, 051903 .

(17) Storm, A. J.; Storm, C.; Chen, J.; Zandbergen, H.; Joanny, J.-F.; Dekker: C. Nano Lett. 2005, 5, 1193-1197.

(18) Fologea, D.; Uplinger, J.; Thomas, B.; McNabb, D. S.; Li, J. Nano Lett. 2005, 5, 1734-1737.

(19) Howorka, S.; Cheley, S.; Bayley, H. Nat. Biotechnol. 2001, 19, 636-639. (20) Astier, Y.; Braha, O.; Bayley, H. J. Am. Chem. Soc. 2006, 128, 17051710 .

(21) Harrell, C. C.; Kohli, P.; Siwy, Z.; Martin, C. R. J. Am. Chem. Soc. 2004, $126,15646-15647$.

(22) Nilsson, J.; Lee, J. R. I.; Ratto, T. V.; Létant, S. E. Adv. Mater. 2006 $18,427-431$. 


\section{Local reduction of membrane thickness}

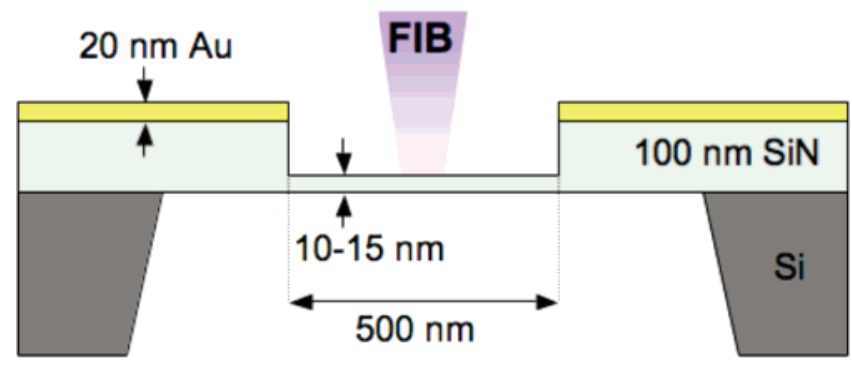

\section{Drilling an initial nanopore}

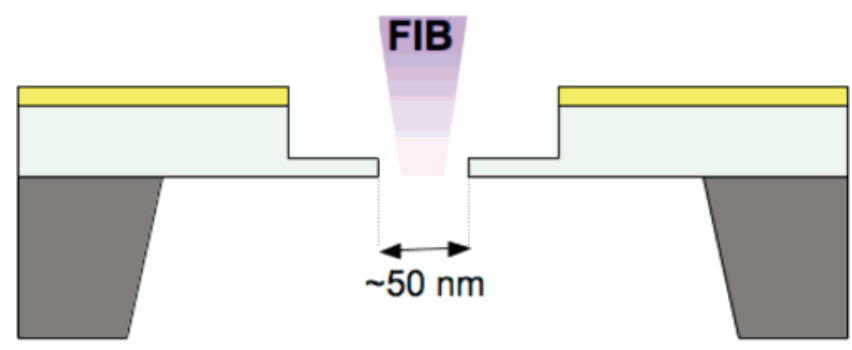

\section{Site-selective silicon oxide deposition}

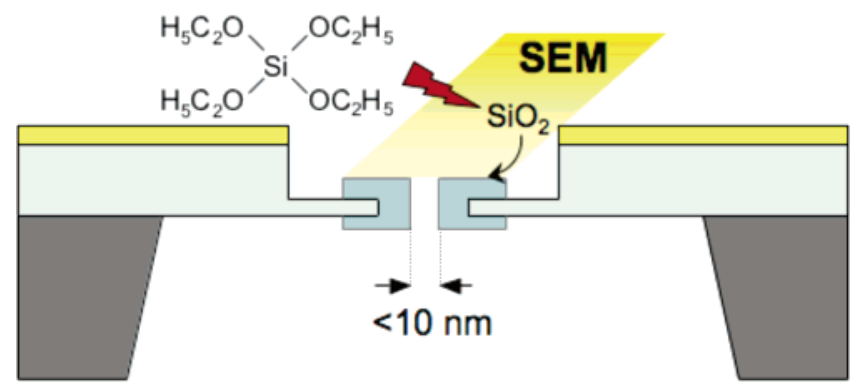

Figure 1. Fabrication and local functionalization of a nanopore in silicon nitride membranes.

which are suited for further functionalization with biomolecules that directly interact with the translocating polymers. Importantly, the capture probes must be localized in the interior or in the vicinity of the pore in order to create a high local concentration of analytes and to exclude undesirable binding to the entire surface.

Here, we present a simple technique for the fabrication of a functional single nanopore that satisfies all of the requirements for efficient and versatile (bio)chemical modifications. The central features of our approach are (i) the use of a low-stress $\mathrm{Si}_{x} \mathrm{~N}_{y}$ membrane, (ii) direct visualization to control the final pore diameter, (iii) local silicon dioxide $\left(\mathrm{SiO}_{2}\right)$ deposition with subnanometer accuracy, and (iv) the prevention of ion contamination during the reduction of the pore size. The procedure for nanopore fabrication described in this work is illustrated in Figure 1. In a circular region of $500 \mathrm{~nm}$ diameter, the 100 -nm-thick low-stress $\mathrm{Si}_{x} \mathrm{~N}_{y}$ membrane is first thinned to $10-15 \mathrm{~nm}$ by FIB milling. Subsequently, a $\sim 50 \mathrm{~nm}$ pore is drilled in the previously thinned membrane using FIB. The final pore diameter is achieved by sequential silicon oxide deposition using the electron beam. The electron beam decomposes the adsorbed precursor tetraethyl orthosilicate $\left(\mathrm{Si}\left(\mathrm{OC}_{2} \mathrm{H}_{5}\right)_{4}, \mathrm{TEOS}\right)$ within a small area around the pore to solid silicon oxide and volatile organic compounds. The evolution of pore diameter is controlled in real time by scanning electron microscope (SEM) imaging.

\section{Experimental Section}

Focused Ion Beam. Low-stress 100-nm-thick $\mathrm{Si}_{x} \mathrm{~N}_{y}$ membranes were fabricated using standard procedures such as low-pressure chemical vapor deposition, photolithography, and anisotropic $\mathrm{KOH}$ etching to provide a $100 \times 100 \mu \mathrm{m}^{2}$ free-standing $\mathrm{Si}_{x} \mathrm{~N}_{y}$ membrane.

For the fabrication of the nanopores, a dual-beam Nova 600 NanoLab (FEI Company) instrument was used. The principle of a dual-beam instrument is described in more detail elsewhere. ${ }^{23}$ The FEI FIB instrument was equipped with a liquid metal ion source providing the $\mathrm{Ga}^{+}$ions used for the sputtering process. First, the 100 -nm-thick membrane was locally thinned to $10-15 \mathrm{~nm}$ using the standard FIB technique (Figure 1). To minimize charging effects due to ion implantation and the formation of secondary electrons, the top side of the membrane was covered with a 20 -nm-thick gold coating that was removed by treatment in potassium iodide before carrying out electrical measurements. Additionally, the sample was irradiated with the electron beam during the sputtering process in order to compensate for surface charging effects. Applying an electron current 10 times higher than the ion current leads to sufficient surface discharging. In a second step, a single small hole with a diameter of 40 to $80 \mathrm{~nm}$ was drilled in the thinned membrane using FIB. To achieve a sufficient small beam diameter, the experiments were carried out with the ion acceleration voltage and current set to 30 $\mathrm{kV}$ and $10 \mathrm{pA}$, respectively; this results in a nominal beam diameter of $12 \mathrm{~nm}$ (full width at half-maximum). Furthermore, the focus and stigmatism of the ion beam were carefully adjusted, and the quality of the beam was checked by drilling holes in the vicinity of the membrane.

Electron-Beam-Assisted Silicon Oxide Deposition. Four gas inlets were mounted onto the FIB instrument to allow the injection of gaseous precursors into the sample chamber while the ion or electron beam followed the prescribed pattern. The adsorption of specific precursors onto the surface of the substrate led either to ionor electron-induced etching or the deposition of material depending on the introduced precursor. With ion- or electron-induced processes, the local deposition of materials such as $\mathrm{SiO}_{2}$ or platinum could be carried out. The initial pore was locally functionalized by the deposition of silicon oxide using TEOS as a precursor. Typical values of the electron beam acceleration voltage/current $I_{\mathrm{e}}$ were $10 \mathrm{kV} / 130$ $\mathrm{pA}$, resulting in a beam diameter of $3.6 \mathrm{~nm}$. To reduce the deposition rate of TEOS on the substrate, the needle of the gas inlet system was retracted to its standby position during the deposition process. The pressure in the chamber was $\sim 2 \times 10^{-7}$ mbar before and $\sim 1 \times 10^{-5}$ mbar after TEOS gas injection. The temperature of the precursor reservoir was maintained at $27.4{ }^{\circ} \mathrm{C}$ to obtain a stable gas flow. With this temperature and the given geometry, a flow of $8 \times 10^{18}$ molecules $\mathrm{s}^{-1}$ was established (Steve Reyntjens, personal communication). Additional $\mathrm{H}_{2} \mathrm{O}$ was introduced into the sample chamber to enhance TEOS decomposition and electron-induced $\mathrm{SiO}_{2}$ deposition.

Electrical Measurements. The nanostructured chip was mounted horizontally in a poly (dimethylsiloxane) chamber located in a Faraday cage with the Si substrate exposed to the top compartment and the free-standing $\mathrm{Si}_{x} \mathrm{~N}_{y}$ membrane containing the nanopore oriented in a fluid-exchange channel (bottom compartment). The nanoporebased chamber was exposed for $30 \mathrm{~s}$ to oxygen plasma (Harrick Scientific Corporation, NY) prior to symmetrical injection of freshly degassed and buffered saline solution $(1 \mathrm{M} \mathrm{NaCl}, 10 \mathrm{mM}$ Tris- $\mathrm{HCl}$, $\mathrm{pH}$ 7.4) in order to facilitate pore wetting. Both compartments were connected to the external measuring circuit using $\mathrm{Ag} / \mathrm{AgCl}$ electrodes. The electrode in the bottom compartment was grounded, whereas the electrode in the top reservoir was connected to the head stage of an Axopatch 200B amplifier (Axon Instruments, Foster City, $\mathrm{CA}$ ) in the voltage clamp mode. The applied voltage refers to the potential in the top compartment relative to the ground potential in

(23) Giannuzzi, L. A.; Stevie, F. A. Introduction to Focused Ion Beams: Instrumentation, Theory, Techniques, and Practice; Springer: New York, 2005. 

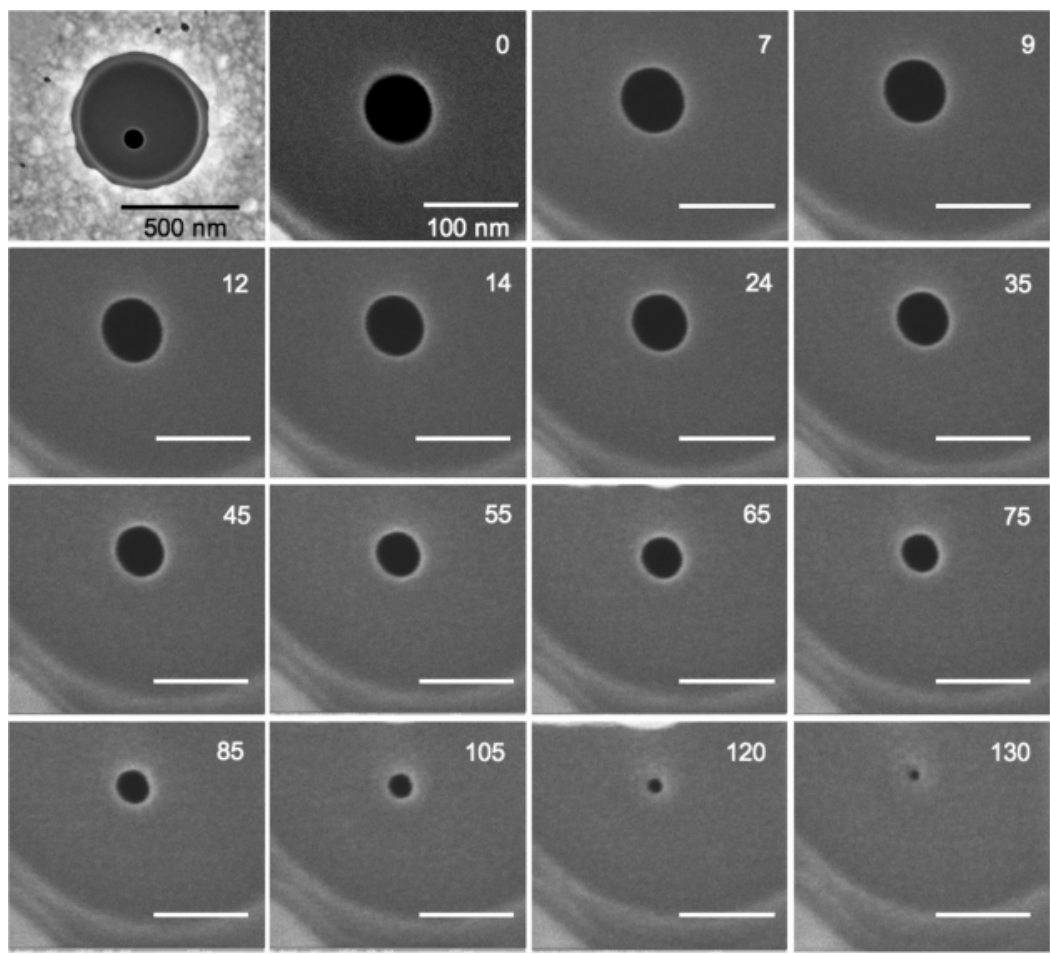

Figure 2. Sequence of scanning electron micrographs demonstrating the controlled size reduction of a nanopore of $80 \mathrm{~nm}$ initial diameter. The initial pore was drilled in a 15-nm-thick $\mathrm{Si}_{x} \mathrm{~N}_{y}$ membrane using the FIB technique prior to local silicon oxide deposition in the presence of TEOS as a precursor gas. The thinned membrane is the 500-nm-diameter circular area seen in the upper left picture. The total number of scans is indicated. Scanning conditions were $t_{\mathrm{d}}=1 \mu \mathrm{s}, n_{x}=1024$, and $\mathrm{HFW}=366 \mathrm{~nm}$.

the bottom compartment. The signal was treated with a low-pass Bessel filter at 1 or $10 \mathrm{kHz}$ and recorded on a videotape using a digital-type recorder (DTR-1204; Biologic, Claix, France). All experiments were performed at room temperature.

\section{Results and Discussion}

Nanopore Fabrication. Figure 2 shows a typical sequence of scanning electron micrographs demonstrating the controlled size reduction of an $80 \mathrm{~nm}$ pore in a $10-15-\mathrm{nm}$-thick $\mathrm{Si}_{x} \mathrm{~N}_{y}$ membrane by the deposition of silicon oxide. The oxide has been generated using electron-beam-induced decomposition of adsorbed TEOS. The deposited $\mathrm{SiO}_{2}$ film is restricted to the area that is irradiated by the focused electron beam resulting in a local functionalization of the substrate around the nanopore. The pore diameter is gradually reduced with successive electron scans at a rate of 0.5 $\mathrm{nm}$ per scan, which allows fine tuning of the nanopore size by simultaneous real-time SEM imaging. Figure 3 depicts the pore diameter as a function of the electron dose $D$, which is related to a single scan by

$$
D=I_{\mathrm{e}} \frac{n_{x}{ }^{2} t_{\mathrm{d}}}{\mathrm{HFW}^{2}}
$$

Here, $t_{\mathrm{d}}$ is the dwell time, $n_{x}$ is number of pixels in the horizontal direction, and HFW is the horizontal field width. Thus, the total dose can be calculated by knowing the number of single scans. We performed experiments at two different electron doses per scan: $D=1.0 \mathrm{nC} \mu \mathrm{m}^{-2} \mathrm{scan}^{-1}$ (high dose) and $8.7 \times 10^{-2} \mathrm{nC}$ $\mu \mathrm{m}^{-2} \operatorname{scan}^{-1}$ (low dose) (Figure 3). For both conditions, the pore diameter decreases linearly with the number of scans, or total dose, indicating that proximity effects do not play an important role even below $10 \mathrm{~nm}$. Moreover, the pores do not suddenly close below a threshold diameter. Proximity effects are mainly related to the formation of secondary electrons. ${ }^{24}$ With $\sim 15$ nm-thick $\mathrm{Si}_{x} \mathrm{~N}_{y}$ membranes, the generation of secondary electrons

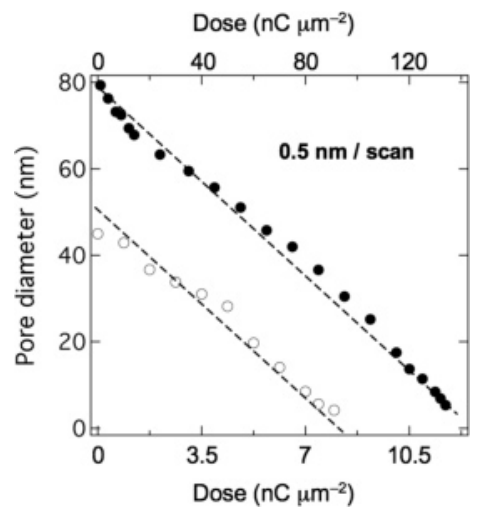

Figure 3. Diameter of nanopores as a function of the dose for two individual experiments. For the initial $80 \mathrm{~nm}$ pore (filled circles, top axis), the scanning parameters are the same as in Figure 2, resulting in a single scan dose of $D=1.0 \mathrm{nC} \mu \mathrm{m}^{-2} \mathrm{scan}^{-1}$. The maximum dose corresponds to the dose of 134 successive scans. For the initial $45 \mathrm{~nm}$ pore (empty circles, bottom axis), the scanning parameters are $t_{\mathrm{d}}=0.3 \mu \mathrm{s}, n_{x}=512$, and $\mathrm{HFW}=343 \mathrm{~nm}$, leading to a single scan dose of $D=8.7 \times 10^{-2} \mathrm{nC} \mu \mathrm{m}^{-2} \mathrm{scan}^{-1}$. The maximum dose corresponds to a dose of 91 successive scans. The doses per scan have been calculated using eq 1 . Both dashed lines have an equal slope of $0.5 \mathrm{~nm} \mathrm{scan}{ }^{-1}$.

is dramatically lowered and accompanied by a reduction in proximity effects. A constant reduction of the pore diameter at a rate of $0.5 \mathrm{~nm}$ per scan has been measured, which allows precise control of the final nanopore diameter. This value was highly reproducible over 25 fabricated nanopores.

The deposition rate remains constant for the different scan parameters that we used in our experiments. Electron-induced decomposition of the precursor is a heterogeneous process that occurs on the surface of the substrate. Thus, the deposition rate

(24) Dobisz, E. A.; Marrian, C. R. K.; Salvino R. E.; Ancona, M. A.; Perkins F. K. J. Vac. Sci. Technol., B 1993, 11, 2733-2740. 
is proportional to the surface coverage $\theta$ of the precursor molecules. ${ }^{25}$ The change in $\theta$ can be written as

$$
\frac{\partial \theta(t)}{\partial t}=\frac{g F}{N_{0}}(1-\theta(t))-\frac{1}{\tau_{\mathrm{des}}} \theta(t)-\frac{m s J(t)}{N_{0}} \theta(t)
$$

where $F$ is the precursor gas flow, $g$ is the sticking probability, $m$ is the number of precursor molecules participating in the reaction, and $s$ is the reaction yield. $J(t), N_{0}$, and $\tau_{\text {des }}$ are the current density, the number of active surface sites and the desorption constant, respectively. The first term in eq 2 corresponds to adsorption, the second to spontaneous desorption, and the third to electron-induced reaction. In the absence of the electron beam, the third term of eq 2 vanishes and the surface coverage increases because of precursor adsorption. In the presence of the focused electron beam, the third term of eq 2 becomes dominant, and the irradiated area is depleted in a very short time. After depletion of the pixel area, the conversion rate of TEOS to silicon oxide depends only on the adsorption rate of TEOS, which is slow compared to the decomposition of the adsorbed precursor. Therefore, above a certain dose $D$ per scan, the amount of silicon oxide deposited per scan becomes insensitive to the dose and thus constant. The elapsed time between two consecutive scans is on the order of $1 \mathrm{~s}$, thus precursor molecules can be reapplied to the surface before a subsequent scan converts them into deposited silicon oxide. As a result, the electron-beaminduced deposition of silicon oxide can be well controlled. Because the deposition rate per scan in our experiments does not depend on the dose per scan, we conclude that for a single scan the average irradiation time of a pixel is long enough to decompose all precursor molecules adsorbed within the pixel area.

The thickness $d$ of a formal $\mathrm{SiO}_{2}$ monolayer can be estimated using its density $\rho$ and molecular weight $M$

$$
d=\sqrt[3]{\frac{M}{\rho_{0} N_{\mathrm{A}}}}
$$

where $M=60.08 \mathrm{~g} \mathrm{~mol}^{-1}, \rho_{0}=2.5 \mathrm{~g} \mathrm{~cm}^{-3}$, and $N_{\mathrm{A}}$ is Avogadro's number. Equation 3 predicts the thickness of a formal $\mathrm{SiO}_{2}$ monolayer to be $0.34 \mathrm{~nm}$. This simple calculation shows that at the given pressure of precursor molecules, silicon oxide can be sequencially deposited monolayer by monolayer, similar to atomic layer deposition. This allows very precise control of the final pore diameter.

The decomposition of TEOS as a precursor for $\mathrm{SiO}_{2}$ deposition can also be performed using a FIB..$^{22,26}$ Very recently, Nilsson et al. ${ }^{22}$ achieved localized shrinking and functionalization of 500- to 1000-nm-diameter holes with oxide deposition by FIBinduced decomposition of TEOS. The oxide ring surrounding the pore can be modified with standard silane chemistry, showing the usefulness of the approach for confined functionalization. However, electron-beam-assisted deposition offers a number of major advantages over the FIB technique: (i) Real-time imaging of the pore diameter by SEM allows in situ feedback for growth control. (ii) The absence of gallium contamination ensures that the pore surface is highly insulating. The resistivity of deposited silicon oxide films is 2 orders of magnitude higher using the electron-beam-induced deposition technique ${ }^{26}$ than when using FIB. This is crucial for reproducible and low-noise electrical measurements of nonrectifying nanopores. ${ }^{10}$ (iii) The deposition rate is slower, ${ }^{26}$ which leads to better control of the final pore

(25) Edinger, K.; Kraus, T. J. Vac. Sci. Technol., B 2000, 18, 3190-3193. (26) Lipp, S.; Frey, L.; Lehrer, C.; Frank, B.; Demm, E.; Pauthner, S.; Ryssel, H. J. Vac. Sci. Technol., B 1996, 14, 3920-3923.

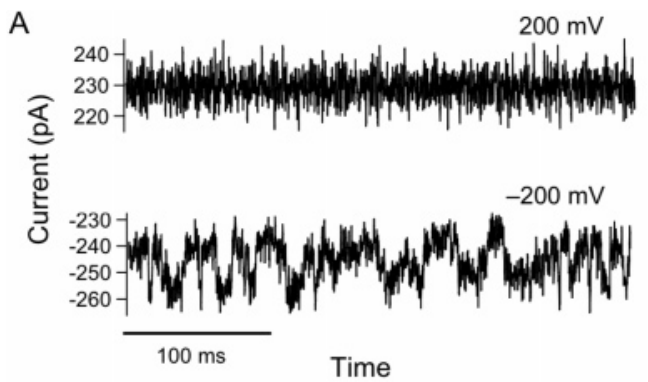

B

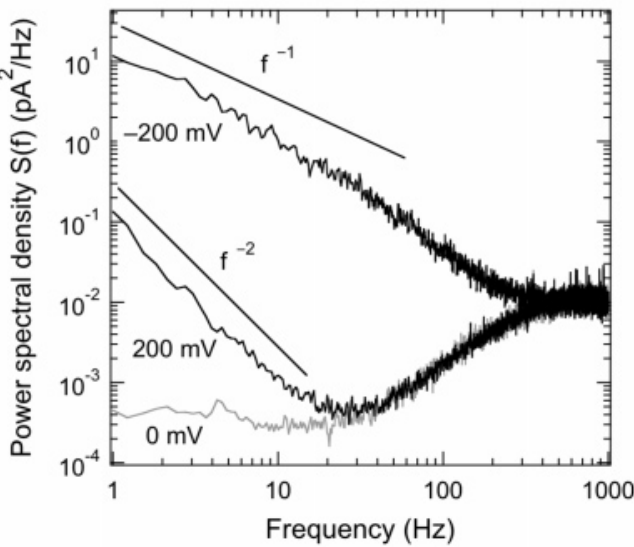

Figure 4. (A) Ion current flow through a single nanopore coated with $\sim 18 \mathrm{~nm}$ silicon oxide with a final pore diameter of $\sim 4 \mathrm{~nm}$ and a total channel length of $\sim 35 \mathrm{~nm}$. The applied voltages were 200 $\mathrm{mV}$ (upper curve) and $-200 \mathrm{mV}$ (lower curve). The bathing solution was $1 \mathrm{M} \mathrm{NaCl}$ and $10 \mathrm{mM}$ Tris-HCl, $\mathrm{pH}$ 7.4. (B) Low-frequency spectral densities of the nanopore currents. The gray curve is the background noise recorded at zero voltage. The low-frequency component of the spectra can be fitted using an $f^{-2}$ function for positively voltage biased pores and an $f^{-1}$ function for negatively voltage biased pores. The bandwidth was $1 \mathrm{kHz}$, and the sampling frequency was $10 \mathrm{kHz}$.

diameter. The diameter of the pores can be decreased monolayer by monolayer and thus controlled very precisely. (iv) With the electron beam, the deposition rate becomes independent of the dose per scan above a certain threshold value of $D$. In contrast, an ion beam leads to the removal of material, and no deposition takes place.

Energy-dispersed X-ray analysis revealed carbon contamination, which might arise from imperfect decomposition of TEOS and carbon residues originating from organic contaminants on the membrane. To reduce organic surface contaminants, the samples were cleaned by oxygen plasma treatment prior to the FIB experiments. In addition, the sample chamber was pumped overnight before starting the experiments. The concentrations of carbon found in silicon oxide are similar for ion and electron beam depositions. ${ }^{26}$

TEOS adsorbs on both sides of the membrane. The electrons have enough energy to penetrate across the membrane, leading to silicon oxide deposition also on the side of the membrane that is not directly exposed to the electron beam (back side). The deposition of silicon dioxide produces nanochannels with a final length exceeding the pore diameter. Although short nanopores provide a higher sensitivity for the electrical detection of permeating analytes (the relative amplitude of current decrease is inversely proportional to the channel length), the ability of long nanochannels to detect single molecular events has been demonstrated. $^{7}$

Ionic Current Characterization. Figure 4A shows typical current recordings obtained from a single 40-nm-diameter and 
10-nm-long initial pore that has been functionalized with $\sim 18$ $\mathrm{nm}$ silicon oxide on the top side of the membrane. Assuming that TEOS adsorption on the back side of the membrane leads to $\sim 7$ $\mathrm{nm}$ silicon oxide deposition, the final pore diameter and length are approximately 4 and $35 \mathrm{~nm}$, respectively. The estimated resistance $R$ of a cylindrical nanopore for freely diffusing ions (no interaction with the channel surface) is ${ }^{27,28}$

$$
R=\rho\left(\frac{L}{\pi f_{\mathrm{c}} r_{\text {eff }}^{2}}+\frac{1}{2 r_{\text {eff }}}\right)
$$

Here, $\rho$ is the bulk resistivity, $r_{\text {eff }}$ is the effective radius of a nanopore, $L$ is its length, and $f_{\mathrm{c}}$ is the Faxen correction factor, which accounts for frictional interactions between the permeating ions and the channel walls. Using $\rho=10.5 \Omega \mathrm{cm}(1 \mathrm{M} \mathrm{NaCl}$ solution), $r_{\mathrm{eff}}=2 \mathrm{~nm}, L=35 \mathrm{~nm}$, and $f_{\mathrm{c}}=0.9$, which corresponds to an ion to pore radius ratio of 0.05 , a nanopore conductance of $2.8 \mathrm{nS}$ is calculated. This value is approximately 2 times larger than the $\sim 1.2 \mathrm{nS}$ conductance experimentally found. A similar restriction on passive ionic transport at high salt concentration has been observed in large water-filled biological channels ${ }^{28}$ and in solid-state nanopore ${ }^{29}$ and is attributed to surface charge effects. Although the exact thickness of silicon oxide deposited on the back side of the membrane is not known, the result is valid for any reasonable values of the channel length.

There is an asymmetry in channel conductance with the sign of the applied voltage. The averaged values of the currents are about $230 \mathrm{pA}$ at $200 \mathrm{mV}$ and $-250 \mathrm{pA}$ at $-200 \mathrm{mV}$ (Figure 4A). More pronounced is the asymmetry in current fluctuations with the sign of the applied voltage. In contrast to the relatively smooth current curve for positive voltages, current fluctuations, corresponding to a change in conductance of several hundred picosiemens, have been observed with a negative voltage. Stepwise current transitions occur over a wide range of time scales and were observed at all studied voltages (from -50 to $-800 \mathrm{mV})$.

Averaged power spectral densities $S(f)$ of current noise were calculated for different voltages over 10-20 segments of $10 \mathrm{~s}$ corresponding to 100000 points for each segment. Figure 4B shows that the low-frequency amplitude of current noise is 2 orders of magnitude higher at $-200 \mathrm{mV}$ than at $200 \mathrm{mV}$. Moreover, the open nanopore spectrum behaves as $f^{-2}$ in the frequency range of $1<f<10 \mathrm{~Hz}$ at positive voltage, whereas stepwise conductance fluctuations observed at negative voltage lead to $f^{-1}$ behavior in the frequency range of $1<f<200 \mathrm{~Hz}$.

The $f^{-\alpha}$ flicker noise with $\alpha=1$ or 2 has already been observed in biological ${ }^{30,31}$ and artificial channels. ${ }^{10,32}$ Here we show that

(27) Hille, B. Ionic Channels of Excitable Membranes, 3rd ed.; Sinauer: Sunderland, MA, 2001.

(28) Danelon, C.; Suenaga, A.; Winterhalter, M.; Yamato, I. Biophys. Chem. 2003, 104, 591-603.

(29) Ho, C.; Qiao, R.; Heng, J. B.; Chatterjee, A.; Timp, R. J.; Aluru, N. R.; Timp, G. Proc. Natl. Acad. Sci. U.S.A. 2005, 102, 10445-10450.

(30) Wohnsland, F.; Benz, R. J. Membr. Biol. 1997, 158, 77-85.

(31) Bezrukov, S. M.; Winterhalter, M. Phys. Rev. Lett. 2000, 85, 202-205. each behavior can be observed on an individual nanochannel at a specific polarity of the applied voltage. Thus, our device is attractive for investigating charge-transport phenomena in confined systems. The $f^{-1}$ noise reflects equilibrium conductance fluctuations caused by structural dynamics of the channel wall constituents. ${ }^{31,32}$ The generation of this noise in our nanopores may be related to reversible interactions between the permeating ions and the negative surface charges distributed on the silicon oxide layer constituting the pore walls. The origin of voltageinduced current noise asymmetry can be assigned to an asymmetric potential energy profile for potassium ions interacting with permanent negative surface charges of the pore. The asymmetry may be caused by the fact that one side of the membrane surrounding the nanopore has been preferentially coated with silicon oxide or by a slightly asymmetric radius profile along the channel axis. ${ }^{33}$

\section{Conclusions}

We have presented a simple procedure for the fabrication of single nanopores that are locally functionalized with silicon oxide by electron-beam-induced deposition. Because of successive monolayer-by-monolayer deposition of silicon oxide, the final diameter of the pores could be controlled with subnanometer resolution. Furthermore, the silicon oxide is suited for further biochemical surface modifications. Electrical characterization of the nanopores revealed the $f^{-1}$ behavior of the noise at negative voltage, which originates from slow conductance fluctuations of the open pore.

The results suggest that the use of our nanopores for analyte sensing is recommended at positive voltage, where low-frequency current noise does not interfere with analyte-specific current fluctuations. Artificial nanopores have been widely used for nucleic acid detection. ${ }^{7,8,10-18}$ The length of our channel largely exceeds the size of single bases, precluding sequencing application. However, the $\mathrm{SiO}_{2}$-coated nanochannel offers promising features for further biochemical functionalization with silane chemistry. Oligonucleotides or DNA-binding proteins can be attached in the pore vicinity to capture single DNA molecules. Moreover, the diameter of the pore can be adjusted (initial diameter and thickness of silicon oxide) to allow the transport of larger objects such as nanoparticles, colloids, ${ }^{34}$ or immune complexes $^{35}$ for high-resolution binding assays.

Acknowledgment. This work was supported by the European Commission via contract LSHG-CT-2004-504601 (E-MeP). We thank Dr. Raissa Trend for carefully reading the manuscript and Dr. Marco Cantoni (CIME, EPFL) for his assistance with energydispersed X-ray experiments.

\section{LA061321C}

(32) Siwy, Z.; Fulinski, A. Phys. Rev. Lett. 2002, 89, 158101.

(33) Siwy, Z. S.; Powell, M. R.; Kalman, E.; Astumian, R. D.; Eisenberg, R. S. Nano Lett. 2006, 6, 473-477.

(34) Saleh, O. A.; Sohn, L. L. Proc. Natl. Acad. Sci. U.S.A. 2003, 100, 820824

(35) Uram, J. D.; Ke, K.; Hunt, A. J.; Mayer, M. Angew. Chem., Int. Ed. 2006, $45,2281-2285$ 